\title{
Research of Sonochemistry of the Dairy Systems to Improve the Properties of Milk Semis
}

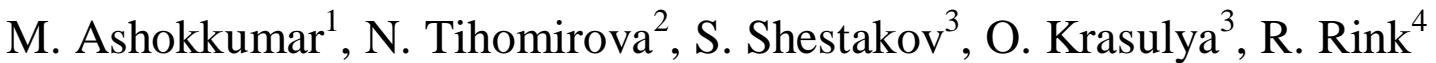 \\ ${ }^{1}$ University of Melbourne, Melbourne \\ ${ }^{2}$ Moscow State University of Food Production, Moscow \\ ${ }^{3}$ Moscow State University of Technology and Management, Moscow \\ ${ }^{4}$ Oil Tech Production OY, Tallinn
}

\begin{abstract}
The paper describes the results of recent research in the field of sonochemistry of the dairy products which was conducted from Australia, Russia, Belarus and Estonia. This work is related to the technology of preparation of composite formula milk from natural milk, vegetable oils and dry milk products with cavitation treatment of water used. She is devoted to obtaining a homogeneous mixtures of dairy semiproducts, from which subsequently produce dairy products such as cottage cheese or cheese, where most of the water is removed with sera. It is shown that sonochemical water treatment has a positive effect on the entire process and its outcome.
\end{abstract}

Keywords: Sonochemistry, water conditioning, dairy semiproducts

\section{INTRODUCTION}

The dairy industry is one of the leading in food industry on the use of artificial hydration, because in it sometimes taken keep milk in dried form and restore to it if necessary by water. Even despite the fact that the water in many foods made from milk is removed in the process of cooking as serum, but it has an important impact on the quality of finished products. The water in the milk, as well as in the composition of fluids that are produced by a living organism, has special properties. Before get to them the water is passes through biological membranes, is disintegrated on the individual molecules, is involved in protein synthesis and increases hydrophilicity of aminoacids. Water used in the restoration of dry dairy ingredients may be close to a state in which it is in natural milk, when the energy of its connection with the protein is maximum. The very same hydration as any reversible chemical reaction in accordance with the law Le Chatelier-Braun is more intense in the energetically favorable for it conditions. Because hydration is - an exothermic process, it happens when the shell of protein is also constructed of individual water molecules, unrelated to the start of the reaction [1].

\section{REVIEW OF THE TECHNICAL AND PATENT LITERATURE}

Application of cavitation in the processing of milk is already known. For example, for this apply a hydrodynamic cavitation in devices rotary-pulsatile type, which make the pasteurization and homogenization of milk. Specialists from the University of Mexico have developed a method of disinfection of milk. It is based on cavitation and does not require heating the entire fluid, but can also replace and pasteurization.

Many useful reactions initiated by ultrasound in the milk, occur on base the mechanisms of the effects on the supramolecular structure of biopolymers [2]. So considers researchers of food sonochemistry from Food Science Australia. But while forming during the pyrolysis of gas mixture in cavitation bubbles free radicals, and also the synthesis and diffusion into liquids the peroxy compounds undesirable. This is established by the authors [3].

Also at the Institute of Chemical Physics RAS named N. Semenov was measured frequently is used now in studies of state moisture in food substances the proton magnetic relaxation in distilled water subjecting its to preliminary processing in a sonochemical reactor. They showed that the spin-spin relaxation with a characteristic time of $T_{2}$ acquires after processing two-component character. Two-component recession clearly observed for 2-3 hours, indicating that the appearance of the water phases with on the other molecular mobility that exists in a limited time. During the time of relaxation this nonequilibrium state, a water and has an anomalous solubility and in this period it is asymptotically returns to equilibrium. From this water is created so dense the hydration shells, which are capable of increasing thermoresistance of proteins [3].

Homogenization of milk (the dispersion of large fat globules) by the cavitation effect is due to selectivity. Particles of the fat of different sizes is destroyed by pulses of pressure is not equally, that in the first place leads to the destruction of largest. In the dispersion process with increasing the surface of separation media there is a deficiency of substances that stabilize the emulsion of milk: proteins, di- and monoglycerides of fatty acids. If proteins can be moving to the surface of fat from the colloidal water phase of milk, a little 
breaking the equilibrium of the entire system, but the di- and monoglycerides in a balanced dispersed system of milk is take nowhere.

Found that on the frequencies of industrial emitters ultrasound cavitation threshold, above which there is a hydrolysis of milk fat, is overcome with the amplitude of the sound pressure is about 3 times higher than the hydrostatic pressure [4]. But together with hydrolysis formed and fatty acid residues (acyl) what increases the acidity and reduce the stability of the resulting mixture. In this work, studies were carried out milk mixtures, synthesized in the cavitation reactor in the absence of hydrolysis of fat. Processing is performed in the mode of hydrolysis of fat (control) and in the mode only disintegrations of structure protein of skimmed milk powder (experience). Relative stability to sedimentation of the samples was measured by the photometric method. For this, the specimens of each of the samples taken from the top and bottom of the beaker after assertion were measured values of the optical transmittance in the green region of the spectrum and calculated their ratio. It was found that at mixtures prepared in the mode of hydrolysis of fat, the optical density is generally higher because they have received is higher dispersion. But a bundle of samples prepared without hydrolysis less because at they excluded the effect on the stability of increased acidity.

Used for homogenization, including milk, ultrasound device by patent CA 2111802. In him is impossible to control the ratio of the hydrostatic and the average sound pressure, as the gap in which the process takes place, is a small. But because of it the energy cost of creating cavitation should be great. Homogenization of milk in [5]. includes processing it through recirculation at temperatures up to $+85{ }^{\circ} \mathrm{C}$ with respect to the acoustic transducer for forming cavitation. But heating the milk is the drawback of this homogenization, because, although the threshold of cavitation in liquids decreases with increasing temperature, but it is accompanied by a reduction of energy same of cavitation [6]. This reduces the formation in milk dual solubility substances which stabilize the emulsion of milk. A rotor-pulsating device used for homogenization also [7]. In such a device as a result of the pressure gradients in the milk of cavitation occurs, which destroys fat phase and makes a uniform particles size of milk fat. By increasing the surface area of the oil phase in the process of homogenization on the surface of the particles fat there a deficiency of substances for stabilizing the emulsion of milk. This can later lead to the inverse process - the coalescence of fat. Hence, the resulting milk formula will not be stable. To increase the stability of the emulsion of milk on the border between the lipid phase and the aqueous medium, which is increases during homogenization, sometimes artificially create the separation shell from proteins [4]. For this purpose before the acoustic cavitation to milk added proteins in amounts proportional to the expected increase in the specific surface of the fat phase. So the mixture is obtained which has a higher protein content. However, the protein itself is not prepared for hydration, because for the disintegration of its structure more mportant component of the energy of cavitation serves kinetic energy. For acoustic cavitation the ratio of energies has the advantage in favor of the potential component [8].

In most of the known methods of hydration of biopolymers through preliminary processing of water by cavitation the number of energy does not explicitly set. For example, in [9], where the water or an aqueous solution before being mixed with the biomass is treated with cavitation, given only the lower limit of the intensity of ultrasound divided by the square of hydrostatic pressure, what defines only the required power. The time activity of cavitation, i.e. the amount of energy for sonochemical changes in water remains arbitrary value. In patent application WO 2007111524 cavitation is cause by radiation of ultrasound with amplitude of sound pressure 5.5 values of static pressure in the cavitation reactor. Energy necessary to destroy the structure of water is given here through productivity of reactor and his electrical capacity. But the duration of the cavitation treatment of water here is established by examining the dependence of the dynamic viscosity the water from the time of activity of the cavitation. It was assumed that the viscosity of water is determined by the ratio of volumes of its structured and unstructured components of water and obeys the law Einstein-Smoluchowsky [10]. However, studies carried out in the University of Leiden [11], suggest that value of process performance is based on this hypothesis is understated. In practice, this can lead to excessive energy consumption.

Generally the epithermal energy transfer makes processes sonochemistry an order of magnitude more economical than thermal. Can compare, for example, the pasteurization, when the whole mass of the liquid containing the bacteria, is heated to a temperature of $+70{ }^{\circ} \mathrm{C}$ and above, which is held certain time and then sometimes and forcibly cooled, with a cavitational bacteriolysis, where for a mechanical destruction shells of microbes is requires only several periods of ultrasonic wave

\section{DISCLOSURE HYPOTHESIS}

Before the authors of the task was to find a way to obtain homogeneous, stable milk mixture with highprotein but without excessive costs on it of acoustic energy.

Known that cavitation with a certain level of potential energy displays water on some time out from the thermodynamic equilibrium. The water on the relaxation time of the nonequilibrium state acquires abnormally high hydration ability with respect to proteins [12]. Dense hydration shells which formed on the containing sulfur active groups of aminoacids of the protein are hinder it from thermal denaturation [13]. Therefore, if 
hydrate shell formed on molecular groups of milk protein, which include sulfur, they not allow $\square$-casein blocking of $\square$-lactoglobulin during further thermal exposure during pasteurization mix that will improve rennet coagulability of mixtures.

In addition, to recovery of milk powder better use water with small temporary hardness. Cavitation allows you to easily translate soluble bicarbonates into insoluble carbonate form, effectively reducing the temporary hardness of water. Mechanism of reaction is based on the destruction by pulses pressure from cavitation hydration shells dissolved and existing as ions bicarbonates $\mathrm{Ca}\left(\mathrm{HCO}_{3}\right)_{2}$ and $\mathrm{Mg}\left(\mathrm{HCO}_{3}\right)_{2}$. That is, it is based on the very same phenomenon, which destroys the supramolecular structure of the water, and promotes the transition of these hardness salts into amorphous colloidal form $\mathrm{CaCO}_{3}$ and $\mathrm{MgCO}_{3}$ [14]

Under natural conditions in the milk the proteins form a supramolecular structure due to the dipoledipole interactions between the molecules of aminoacids. This structure can be destroyed by hydrodynamic cavitation rotary-pulsational apparatus. So the aminoacids may be is prepared to join in hydration reaction. The polar centers of aminoacid more active than with molecular associates of water interact with dipoles, which are individual water molecules. But for that the water itself which is also has even at high temperatures own structure, to be unstructured. It is known that the best way to destroy the structure of water, that is, preparation for hydration - sonochemical processing of its in cavitation reactor [12]. Therefore, in order to obtain a positive result, it is sufficient to connect the inlet of mixer dry milk components and water to outlet reactor for water processing. Since the anomalous properties of water is saved did not last long, then at continuous preparing a mixture in apparatus, which is contain a reactor, there is need to harmonize the processing speed of the whole mixture in a rotor-pulsating device with sonochemical processing of water in that reactor. The latter should have the performance depending on the energy used. But, that determine this value necessary to know the specific (per unit volume) energy sonochemical treatment.

In monograph [15] shows a fact of availability the maximum of the function of output of hydrogen peroxide at sonolysis of water, which is explained by the scattering of the acoustic energy on the internal friction in the water to form a heat, which weakens the cavitation. Consequently, the function of disintegration of the structure of the water from the spent energy must also have a maximum. Therefore, the range of energy sonochemical processing can be found through practical optimization. This range will be common to all the reactors in accordance with the principle of similarity of cavitation processes [8]. It is clear that have in mind the acoustic energy, because there are some ways to convert electrical energy into the energy of elastic vibrations with different coefficients of performance. In the device from patent application WO 2007111524 is used a way to convert due to the inverse piezoelectric effect. Efficiency it is $90 \%$, and the specific performance of the device $0,6 \mathrm{~m}^{3} / \mathrm{h}:(4 \mathrm{~kW} \cdot 0,9)=0,17 \mathrm{~m}^{3} / \mathrm{KW} \cdot h$ Reactor which Is used here in practical optimize with power capacity of 630 watts is powered by a magnetostrictive transducer (efficiency $50 \%$ ), has a productivity of about $2 \mathrm{dm}^{3} / \mathrm{min}$.

\section{EXPERIMENTAL CONFIRMATION OF THE HYPOTHESIS}

Optimization was carried out using of that reactor in recirculation mode on the stand (Fig. 1), consisting of the dispersant 2 , in as was which used a laboratory pump-emulsifier, mixer 3 , filled before each experiment of whole milk by three quarters. The feed rate of water into the mixer is always maintained constant. For this used stabilizer 4, consisting of a coaxially placed one inside the other tanks and choke 5 which was coaxially mounted on the pipe coming out of them and adjusted on the minimum flow rate in the experiment. Skimmed milk powder is introduced, just sprinkling it in the mixer 3. Into the internal tank of the stabilizer from the outlet of the reactor through a three-way valve 6 is continuously fed water after sonochemical process. Performance adjusted through the setting on the output of the reactor the choke 7 and measuring cup. Thus, the reactor can run at any given performance, but the water from the stabilizer into the mixer is always fed at the same rate. Excess water at the filling of the tank flows through the drain pipe 8 which mounted on the bottom of the outer container. In the mixer, which is being filled during each experiment to the mark 9 for effective the mechanical agitation was set impeller of agitator 10. That is, mixing container had the appearance tank for pasteurizing or fermenting which used in dairy industry. 


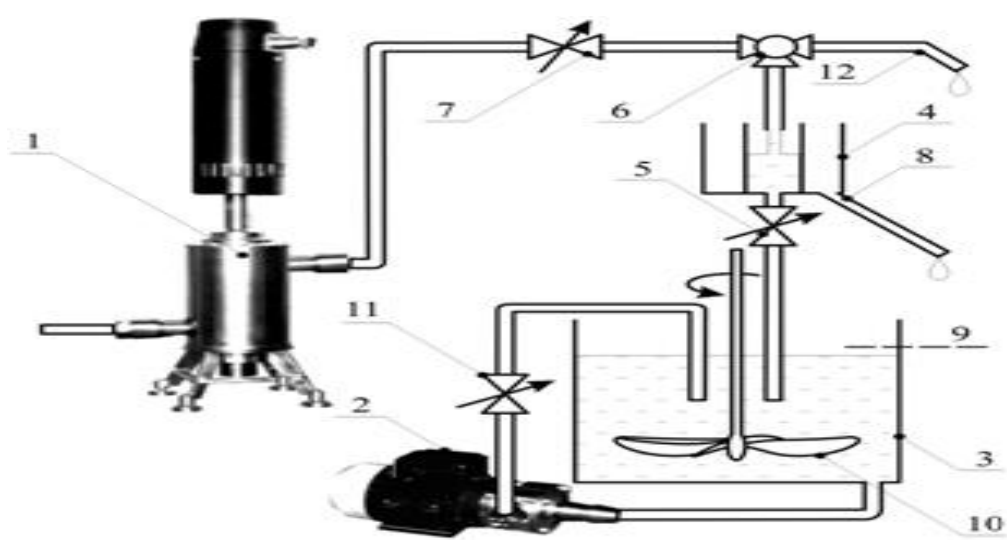

Fig. 1. Scheme of the experimental stand, on which was carried out of the practical optimize of the getting mixture: 1 - cavitation reactor; 2 - a dispersant; 3 - mixer; 4 - stabilizer; 5 - choke; 6 - three-way valve; 7 - choke; 8 - drain connection, 9 - a mark, 10 - impeller mixer, 11 - choke, 12 - drain pipe

The mixer was included in the scheme of recirculation through dispersant mixture with a given of throttle 11 speed. The experiment was conducted at room temperature. Five samples of mixtures were prepared with different productivities of the reactor. To do, when the reactor was off and the three-way valve 6 was switched to the discharge of water through the drain pipe 12, to establish the desired performance was used measuring cup. The mixers perfuse whole milk and add accordance in it with the patent application WO 2007111524 skimmed milk powder, include the impeller of mixer, the dispersant and through 5 seconds switched the three-way valve on the supply of processed water to the stabilizer. By filling out the mixer to the mark 9 three-way valve 6 is switched to for discharge of water through the drain pipe, i.e. stopped supply to the reactor, turn off reactor, and continue mixing and dispersing for another 20 minutes.

The viscosity was measured by rotational viscometer in the volumes taken from the central parts of the tanks for storage of samples after settling for three days at a temperature $+8{ }^{\circ} \mathrm{C}$. The results of these measurements were divided by the viscosity of whole milk with the same number of the skimmed milk powder and the water, but without sonochemical processing. The received discretes regularities of the relative viscosity on the speed of processing were approximated by second-order polynomial the method of least squares. The point corresponding to performance $0.12 \mathrm{~m}^{3} / \mathrm{h}$ lying on the branch of a parabola on the other side from maximum correspond abscissa $0.3 \mathrm{~m}^{3} / \mathrm{h}$. Inside thus obtained the range of viscosity of milk and, consequently, the dispersion of his fat phase, more than at in patent application WO 2007111524. It corresponds to diapason of specific energies of water processing $5 . .99 \mathrm{MJ} / \mathrm{m}^{3}$, which should be shared for cavitation processes and at higher amplitudes of sound pressure [12,15].

\section{FINDINGS. THE PRACTICAL IMPLEMENTATION OF RESEARCH IN INDUSTRY}

Results of this study on an industrial scale can be implemented (Fig. 2) by use as a dispersant rotarypulsational disintegrator (Fig. 3), developed in the homeland of one of the first researchers the mechanical disintegration in rotary apparatus of Dr. J. Hint [16] as well as the sonochemical reactor For example, an industrial processor UIP4000 company Hielscher Systems GmbH [17].

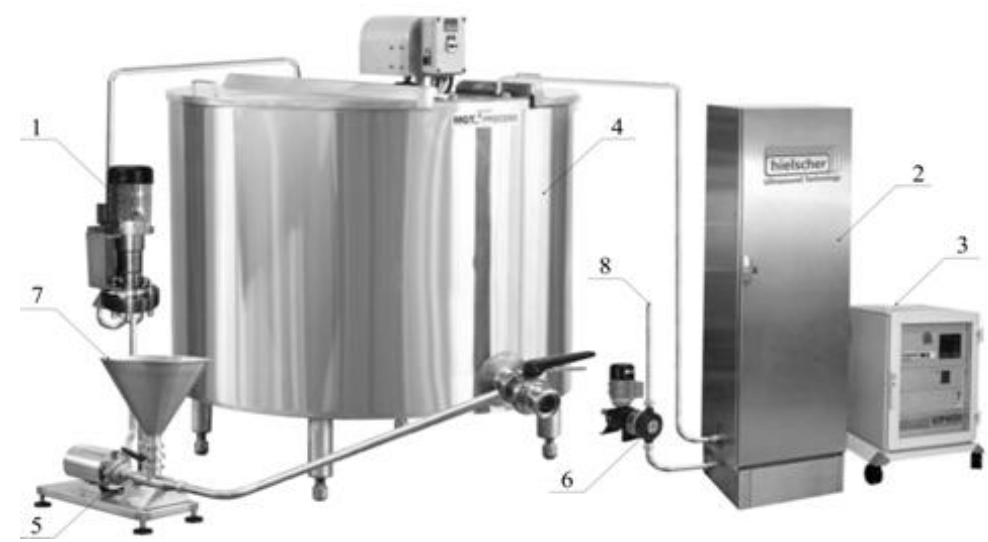

Fig 2. Industrial version of implementation: 1 - rotary dispersant; 2 - sonochemical reactor with generator 3; 4 - mixing tank; 5 - circulation pump; 6 - metering pump; 7 - a device for the introduction of dry skim milk; 8 - water inlet 
This requires uploads dried whole or skim milk or whey powder or dry milk protein concentrate According to patent application WO 2007111524. If the protein may link at hydration to $40 \%$ water by weight [1], need to add on this mass dry milk protein a monomolecular water. It is one third of volume of water which was subjected processing by cavitation [10]. The protein that makes up a structural-mechanical boundary layer on the fat phase, thus will be more hydrated, therefore, will have a greater wettability and surface activity. As the mixing container to make a mixture, can use the ware production of MGT. Rotary disintegrator can include in the recirculation scheme the mixture through this container MGT, include in it a pump SIMPLEX-MS or STAMP-STN and device to boot the skimmed milk powder. From the reactor treated water can be fed directly into the container. For dosing of the water and the setting of the required capacity of the reactor can be used a dosing pump, for example, Pedrollo.

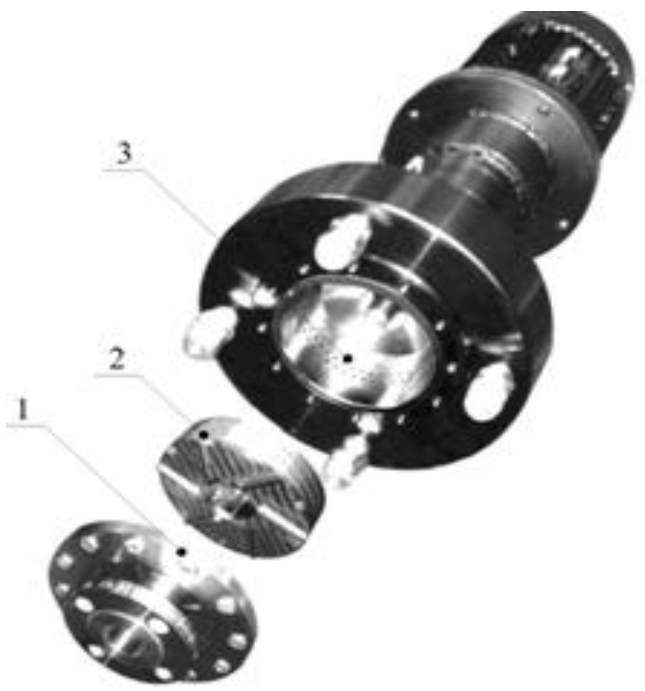

Fig. 3. The dispersant of company Oil Tech Production OY with removed lid of working volume 1 and removed rotor 2 from the stator 3 .

\section{REFERENCES}

[1]. Kuntz I.D. The physical properties of water bound to biomacromolecules. Water relations of foods / Ed. by R.B. Duckworth, (London: Academic Press, 1975)

[2]. Ashokkumar M. at al. The ultrasonic processing of dairy products. Dairy Science and Technology, 2010, V.90, pp. 147-168

[3]. Shestakov S., Krasulya O. Sonochemical technologies in food industry. Electronic Journal "Technical Acoustics", http://www.ejta.org, 2010, 10

[4]. Shestakov S. Technologies of cavitational disintegration in dairy industry. Dairy industry, 2007, 9, pp. 58-60

[5]. Glaznev N. et al. Patent RU 2172107, 2000

[6]. Knapp R., Daily J., Hammitt F. Cavitation. (New York: McGraw Book Company, 1970)

[7]. Volkov G. et al. Patent RU 2222952, 2002

[8]. Shestakov S., Babak V. Mathematical Model of the Spatial Distributing of Density of Erosive Power of Multibubble Cavitation. Applied Physics Research, 2012, Vol. 4, No. 1, pp. 64-77

[9]. Shestakov S. Patent RU 2331478, 2008

[10]. Rogov I., Shestakov S. Epithermal change the thermodynamic equilibrium of water and aqueous solutions: Delusion and Reality. Storage and Processing of Farm Products, 2004, 10, pp. 9-13

[11]. Jinesh K.B., Frenken J.W.M. Experimental evidence for ice formation at room temperature. Physical Review Letters, 2008, 101, 036101

[12]. Shestakov S. Food sonochemistry: concept, theoretical aspects and practical applications. (Saarbrücken: LAMBERT Academic Publishing, 2012)

[13]. Krasulya O. at al. Patent RU 2444201, 2002

[14]. Tikhomirova N. at al. Patent RU 2422371, 2011

[15]. Shestakov S.D. Basics of cavitational disintegration. (Moscow: EVA press, 2001)

[16]. Ashokkumar M. at al. A New Look at Cavitation and the Applications of Its Liquid-Phase Effects in the Processing of Food and Fuel. Applied Physics Research, 2012, V4, 1, pp. 19-29

[17]. Zisu B. et al. Ultrasonic processing of dairy systems in large scale reactors. Ultrasonics Sonochemistry, 2010, 17, pp. 1075-1081 